\title{
Chromospheric activities of late-type stars based on Guoshoujing Telescope
}

\author{
L. Y. Zhang ${ }^{1}$, G. Y. Zhang ${ }^{1}$, J. R. Shi ${ }^{2}$, A. L. Luo ${ }^{2}$, J. K. Zhao ${ }^{2}$, Y. H. \\ Zhao $^{2}$, H. Wu ${ }^{2}$, A. Y. Zhou ${ }^{2}$, Q. F. Pi ${ }^{1}$ and LAMOST Collaboration ${ }^{2}$ \\ ${ }^{1}$ College of Science / Department of Physics, Guizhou University, Guiyang 550025, China \\ email: Liy_zhang@hotmail.com \\ ${ }^{2}$ National Astronomical Observatories, Chinese Academy of Sciences, Beijing 100012, China
}

\begin{abstract}
We present chromospheric activities of late-type stars based on stellar spectrum survey of LAMOST (also called GuoShouJing telescope) in 2010-2013. Using the equivalent widths of the $\mathrm{H}_{\alpha}$ line (larger than $1 \stackrel{\circ}{A}$ ), we have found 6391 active stars from $99741 \mathrm{M}$ stars.
\end{abstract}

Keywords. stars: late-type, stars: activity, stars: chromospheres, stars: spectra, stars: low-mass.

GuoShouJing Telescope (LAMOST) is a Chinese national scientific research facility (Cui et al. 2012; Zhao et al. 2012). We have reduced the stellar spectrum (with a spectral resolution of 1800, Luo et al. 2012) of M candidates of LAMOST in 2010-2013. We measured the chromospheric EW of $\mathrm{H}_{\alpha}$ line using the Hammer program and visually inspected all candidates and manually assigned spectral types (Hawley et al. 2002; Zhang et al. 2012). The criteria to classify active stars are that the $\mathrm{EW}$ of the $\mathrm{H}_{\alpha}$ line is larger than $1 \stackrel{\circ}{A}$, and its error, and the center height of the $\mathrm{H}_{\alpha}$ emission must be 3 times its noise. These are similar to the criteria of West et al. (2011) and Zhang et al. 2012. We found 6391 active stars from $99741 \mathrm{M}$ stars. Figure. 1 shows the LAMOST spectra (left) and the fraction of stars that are active as a function of spectral types (right).
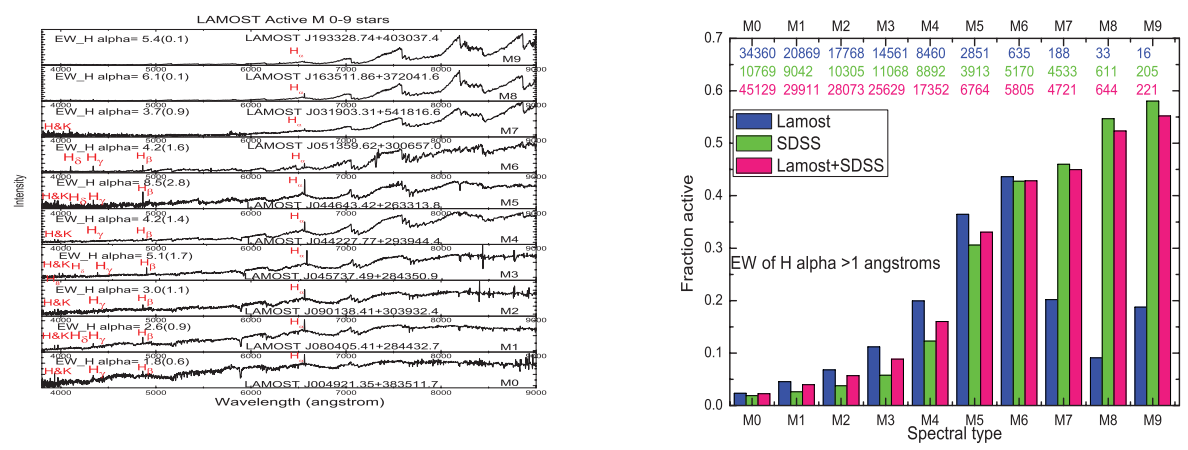

Figure 1. The LAMOST spectra and fraction of active stars as a function of spectral type.

Acknowledgements. This work is supported by the NSFC of No 10978010 and 11263001.

\section{References}

Cui, X. Q., Zhao, Y. H., Chu, Y. Q., et al., 2012, RAA, 12, 1197

Hawley, S. L., Covey, K. R., Knapp, G. R., et al., 2002, AJ, 123, 3409

Luo, A. L., Zhang, H. T., Zhao, Y. H., Zhao, G., et al., 2012, RAA, 12, 1243

West, A. A., Morgan, D. P., Bochanski, J. J., et al., 2011, AJ, 141, 97

Zhao, G., Zhao, Y. H., Chu, Y. Q., et al., 2012, RAA, 12, 723

Zhang, L. Y., Shi, J. R., Zhao, J. K., Luo, A. L., et al., 2012, IAU294, 209 